\title{
Effect of Neural Mobilization Techniques and Nerve Mobilization Exercise on Ulnar Nerve Entrapment Post Supracondylar Humeral Fracture. A Case Report
}

\author{
Ishfaq Bashir Bhat* \\ Department of Physiotherapy, HOSMAT College of Physiotherapy, Bangalore, India \\ *Corresponding Author: Ishfaq Bashir Bhat, Department of Physiotherapy, \\ HOSMAT College of Physiotherapy, Bangalore, India.
}

Received: September 14, 2020

Published: October 08, 2020

(C) All rights are reserved by Ishfaq Bashir

Bhat.

\begin{abstract}
Introduction: The purpose of this case report is to describe the role of ulnar nerve mobilization techniques on post humeral fracture entrapment of ulnar nerve in and around the healing fracture. Ulnar nerve entrapment is the second most of entrapment neuropathy in upper limb. Ulnar nerve can get entrapped in callus in supracondylar fracture.

Case Description: A 22-year-old male student presented to physical therapy, progressing well following supracondylar humeral fracture, sustained 8 weeks prior. During the treatment, the patient reported restricted range of motion and function, with pain. Based on signs and symptoms the patient was having ulnar nerve entrapment.

Results/Outcome: After two weeks of manual therapy (ulnar nerve gliding techniques), and a detailed home exercise program (nerve mobilization exercises and ROM), the patient returned to his normal. There was significant improvement in pain (VAS) and the post treatment McGowan score improved from grade 1 to grade 0 . There was improvement in patient's functions the patient specific functional scale (PSF) score improved from 6 to 1.

Conclusion: This case report describes the effect of neural mobilization techniques in improving ulnar nerve entrapment post supracondylar fracture of humerus. The case report demonstrates improvements and maintaining functional mobility. Based on this report the further research is warranted in this area.
\end{abstract}

Keywords: Neural Mobilization Techniques; Nerve Mobilization Exercise; Supracondylar Fracture; Humerus

\section{Introduction}

Ulnar nerve is one of the three main nerves in upper limb. It arises from brachial plexus. It provides motor and sensory innervation to the upper limb. It originates from ventral rami of $\mathrm{C} 8, \mathrm{~T} 1$ nerve roots.

The nerve travels from medial aspect of arm and forearm and then enters into the wrist and supplies hand and fingers $[1,2]$.

Ulnar nerve provides motor innervation to part of forearm and hand. Ulnar nerve innervates medial part of forearm, wrist and medial one and half digits. It innervates flexor carpi ulnaris, flexor digitorum profundus and flexor digiti minimi brevis.
In hand it provides motor supply to hypothenar muscles, third and fourth lumbricals, dorsal interossei, palmer interossei, adductor pollicis, medial head flexor pollicis brevis, palmaris brevis $[3,4]$.

Ulnar nerve entrapment neuropathy at elbow is the second most common upper extremity neuropathy. The common sites of compression arcade of Struther's ulnar nerve groove, cubital tunnel etc. The ulnar gets compressed due to irritation of due to prolonged stress due to leaning on elbow, fluid build up in elbow, bone spurs, fracture of medial epicondyle, humeral fracture with callus formation, nerve irritation by scar tissue or callus $[5,6]$. 
Effect of Neural Mobilization Techniques and Nerve Mobilization Exercise on Ulnar Nerve Entrapment Post Supracondylar Humeral Fracture. A Case Report

Symptoms of ulnar nerve entrapment include intermittent numbness and tingling along the ulnar border of forearm, fourth and fifth finger, Sensitivity to cold, tenderness in elbow and hand. The physical therapy management for ulnar nerve compression syndromes include impairment based approach which addresses deficits of strength, range of motion and functional goals, the conservative management includes splinting, use of NSAIDs, and nerve gliding protocol $[7,8]$.

Entrapment of ulnar nerve in callus after supracondylar fracture is one of the causes ulnar neuropraxia. Treatment of entrapment neuropraxia can be done by giving ulnar nerve gliding techniques and neve gliding exercises and addressing the strength and functional deficits.

\section{Case Report}

A 22 year old male presented to physiotherapy department with complaints of pain and decreased Range of motion in left elbow after he was managed for supracondylar fracture in his left arm. Eight weeks back the patient fell and on his left arm from his bike the patient was diagnosed with supracondylar fracture in his left arm. On preoperative examination it was revealed that there was no ulnar nerve injury or any other associated injuries. The vascular status was normal with good radial pulse. on radiological examination the fracture was of grade two based on modified Gartland's staging system. The fracture was managed by percutaneous pinning and closed reduction. The pins were removed after four weeks and an $\mathrm{x}$ ray was taken to confirm the healing of fracture and the fracture showed stable fracture alignment and visible callus. On examination there were no signs of pin infection. The patient was initially prescribed ROM exercises in the hospital. Three weeks later the patient was referred to OPD in HOSMAT college of physiotherapy. The patient reported restricted range of motion and pain in elbow. And the patient also reported difficulty in performing functional activities.

On observation and palpation at the site of percutaneous pinning the perforations in skin were healed with no signs of infection. On physical examination it was revealed that there was painful flexion of elbow so the patient was not attempting to complete the elbow flexion beyond 70 degrees. When examiner performed passive range of motion the symptoms were provoked but the end feel was empty. On tapping along the medial aspect of forearm there was tingling sensation along the ulnar border and in the fifth finger (Tinel's sign positive). The upper limb tension test
3 (ULTT3) was positive causing increase in pain and hypesthesia along the distribution of ulnar nerve. No atrophy or any motor loss was found so the patient was classified as Stage 1 one McGowan scoring. The available clinical data suggested the diagnosis of ulnar nerve entrapment.

\section{Intervention}

The intervention consisted of passive ulnar nerve glides ULTT3, range of motion exercises and ulnar nerve gliding exercises as home exercise program. The passive ulnar nerve glides (ULTT3) was performed thrice every week and other exercises were repeated 3 times a day for period of two weeks [9].

The nerve gliding exercises included:

1. Elbow flexion and wrist extension

2. Head tilts with elbow and wrist extension

3. Arm flexion in front of body

4. $\mathrm{A}-\mathrm{OK}$

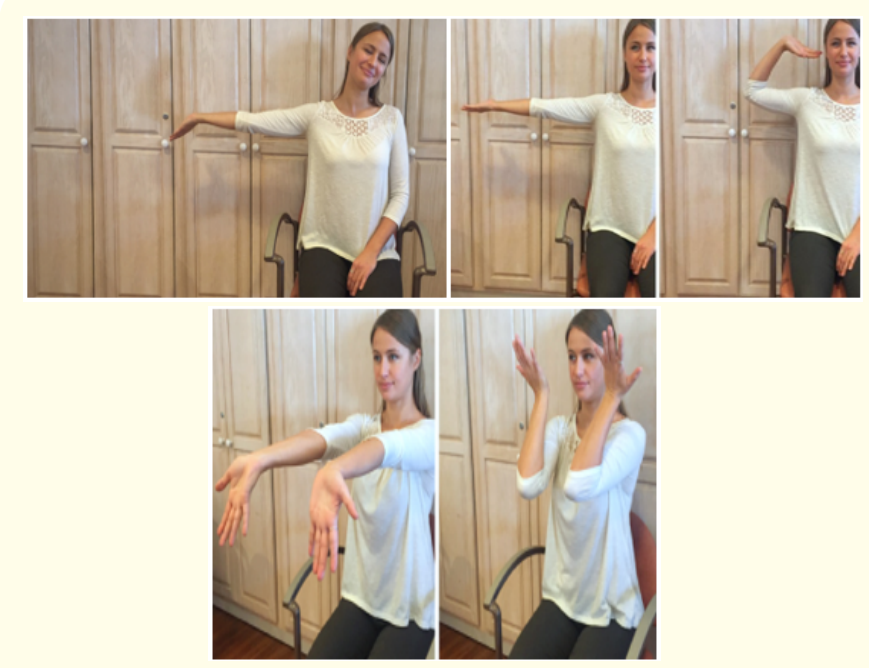

Figure

\section{Outcome measures}

Mcgowans scale as modified by Goldberg MG was used to assess the neurological symptoms of the patient which consists of four grades 1 to $3[10,11]$.

Visual analogue scale (VAS) was used to assess pain in elbow during activities. Pain was assessed as baseline and after treatment and at the end of two weeks. Pain intensity was rated using the VAS, which rates pain on a scale from 0 to 10 , with 0 being no 
pain and 10 being very high pain levels. The visual analog scale has been shown to be a reliable and valid tool for assessing immediate changes in pain intensity, with a test-retest reliability of between $0.95-0.97$ and the minimally clinically important difference of 12 $\mathrm{mm}(+/-3 \mathrm{~mm}$ at a $95 \% \mathrm{CI})[12]$.

Patient specific functional scale was used to evaluate the functional outcomes. It is a useful questionnaire used to quantify activity limitation. The grading scheme is from 0 to 10 for each activity. and total score is sum of activity score divided by number of activities $[13,14]$.

\section{Discussion}

The objective of this study is to show the positive effect of neural mobilization on post fracture humerus where ulnar nerve may became entrapped around the healing fracture. The positive outcome suggests that there are positive benefits of nerve Mobilization techniques on ulnar nerve entrapment post humeral and supracondylar fracture.

It is imperative to consider that other factors also may have positive effects like initiation of range of motion exercises etc.

There is additional need of research on the effect of use of nerve mobilization techniques in ulnar nerve entrapment in general and post humeral fracture ulnar nerve entrapment in particular.

Comparison of pretreatment and post treatment stages using the McGowan classification as modified by Goldberg. The results after treatment showed improvement and the McGowen score was 0 after the end of two weeks.

Patient specific functional scale (PSF) was used to measure the functional outcomes and there was improvement in PSF scores from 6 to 1. At the initial and final assessment, each patient was asked to complete the PSFS. Each patient identified three items that were difficult or they were unable to perform or performed with difficulty, and the degree of difficulty was ranked from 0 to 10 .

Our case report is in line with the study done by Michel W Coppieters, Katrien E Bartholomeeusen, Karel H Stappaerts in which they reported that Incorporated nerve-gliding techniques in the conservative treatment of cubital tunnel syndrome and concluded that movement based management may be beneficial in conservative management of cubital tunnel syndrome [15].
Deran Oskay PT says that use of Neurodynamic Mobilization in the Conservative Treatment of Cubital Tunnel Syndrome may be beneficial for selected patient with mild to moderate symptoms. The treatment included neurodynamic mobilizations including sliding techniques and tensioning techniques which are thought to enhance ulnar nerve gliding and restore neural tissue mobility and it showed long term positive results [16].

Michel W Coppieters, David S Butler in their proposed that possible beneficial effects of nerve gliding exercises on neurophysiological process may assist the clinician in selecting more appropriate nerve gliding exercises in conservative and post operative management of common neuropathies [17].

\section{Conclusion}

This case report describes the effect of neural mobilization techniques in improving ulnar nerve entrapment post supracondylar fracture of humerus. The case report demonstrates improvements and maintaining functional mobility. Based on this report the further research is warranted in this area.

\section{Bibliography}

1. Ramage JL., et al. "Anatomy, shoulder and upper limb". Hand Guyan Canal (2018).

2. Kyle Andrews., et al. "Cubital tunnel syndrome: Anatomy, clinical presentation, and management". Journal of Orthopaedics 15.3 (2018): 832-836.

3. Richard Drake., et al. "Gray's Anatomy for Students". Fourth edition.

4. Human Anatomy by BD Chaurasia's $7^{\text {th }}$ Edition.

5. Dianna Quan MD and Shawn J Bird MD. "Nerve Conduction Studies and Electromyography in the Evaluation of Peripheral Nerve Injuries". The University of Pennsylvania Orthopaedic Journal 12 (1999): 45-51.

6. T Lalanandham and WN Laurence. "Entrapment of the ulnar nerve in the callus of supracondylar fracture of the humerus". Injury 16.2 (1984): 129-130.

7. Svernlov B., et al. "Conservative treatment of the cubital tunnel syndrome". The Journal of Hand Surgery European 34.2 (2009): 201-207.

8. Sahil Kooner., et al. "Conservative treatment of cubital tunnel syndrome: A systematic review". Orthopedic Reviews 11.2 (2019): 7955. 
9. Richard F Ellis., et al. "Neural Mobilization: A Systematic Review of Randomized Controlled Trials with an Analysis of Therapeutic Efficacy". The Journal of Manual and Manipulative Therapy 16.1 (2008): 8-22.

10. McGowan AJ. "The results of transposition of the ulnar nerve for traumatic ulnar neuritis". The Journal of Bone and Joint Surgery British 32-B.3 (1950): 293-301.

11. R Lancigu., et al. "Dellon's anterior submuscular transposition of the ulnar nerve: Retrospective study of 82 operated patients with 11.5 years' follow-up". Chirurgie De La Main 34 (2015): 234-239.

12. Strunce JB., et al. "The immediate effects of thoracic spine fck LR and rib manipulation on subjects with primary complaints of shoulder pain". The Journal of Manual and Manipulative Therapy 17.4 (2009): 230-236.

13. Hefford C., et al. "The patient-specific functional scale: validity, reliability, and responsiveness in patients with upper extremity musculoskeletal problems". Journal of Orthopaedic and Sports Physical Therapy 42.2 (2012): 56-65.

14. Horn KK., et al. "The patient-specific functional scale: psychometrics, clinimetrics, and application as a clinical outcome measure". Journal of Orthopaedic and Sports Physical Therapy 42.1 (2012): 30-D17.

15. Michel W Coppieters., et al. "Incorporating nerve-gliding techniques in the conservative treatment of cubital tunnel syndrome". Journal of Manipulative and Physiological Therapeutics 27.9 (2004): 560-568.

16. Deran Oskay PT., et al. "Neurodynamic Mobilization in the Conservative Treatment of Cubital Tunnel Syndrome: LongTerm Follow-Up of 7 Cases". Journal of Manipulative and Physiological Therapeutics 33.2 (2010): 156-163.

17. Michel W Coppieters and David S Butler. "Do 'sliders' slide and 'tensioners' tension? An analysis of neurodynamic techniques and considerations regarding their application". Manual Therapy 13.3 (2008): 213-221.

\section{Assets from publication with us}

- Prompt Acknowledgement after receiving the article

- Thorough Double blinded peer review

- Rapid Publication

- Issue of Publication Certificate

- High visibility of your Published work

Website: https://www.actascientific.com/

Submit Article: https://www.actascientific.com/submission.php Email us: editor@actascientific.com

Contact us: +919182824667

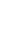

